\title{
Assessment of stone heritage decay by X-ray computed microtomography: II - a case study of Portuguese limestones
}

\author{
Maurício, A. , Figueiredo, C. , Pereira, M.F. , Alves, C. ${ }^{* *}$, Bergounioux, M. ${ }^{* * *}$ and Rozenbaum O. \\ * CERENA/CEPGIST, Instituto Superior Técnico, Universidade de Lisboa, Lisbon, PORTUGAL \\ ${ }^{* *}$ Centro de Investigação Geológica, Ordenamento e Valorização de Recursos - Universidade do Minho, \\ PORTUGAL \\ ****niversité d'Orléans, UFR Sciences, Math., Labo. MAPMO, UMR 6628, Route de Chartres, BP 6759, \\ 45067 Orléans cedex 2, FRANCE \\ *****Institut des Sciences de la Terre d'Orléans (ISTO) UMR7327 CNRS/Université d'Orléans, FRANCE \\ Email: pcd2045@ist.utl.pt
}

X-ray Computed Micro-Tomography or micro-CT ( $\mu-\mathrm{CT})$ is a frequently used nondestructive 3D imaging and analysis technique for the investigation of the stone matrix texture, modal composition, void's spectrum (pores and/or other material's discontinuities). and interconnectivity. These are fundamental characteristics that can be mapped and parameterized and from which other secondary parameters can be deduced and estimated. The accuracy of all these parameters' estimations depends strongly on the quality and robustness of segmentation process used to identify the void's and solid's matrix spectra subspaces. Inherent noise and other artefacts in the reconstructed scanned images coupled with ambient illumination, variation of grey levels within the subspace, inadequate contrast, subjective manual segmentation methods, explains the absence of a single universally applicable automatic segmentation process solution. This case study aims to contribute to better characterise and/or quantify 3D spatial structures (textures) of Portuguese limestones, using a 3D microscopy image analysis method. It follows a preliminary ( $\mu$-XCT-) investigation of sulphate salt degradation of three Portuguese Dimension Stones ("Semi-Rijo-SR", "Moca Creme-MC" and "Travertino-TV", based on the Portuguese Standard NP EN 12370 (2001) [1], [2], because of their relatively high porosity and their almost pure composition. The samples were scanned with an industrial CT device Nanotom 180NF (GE Phoenix|x-ray, Wunstorf, Germany) available at the ISTO, using a 3 to $4 \mu \mathrm{m}$ pixel size resolution. A fundamental step that must be considered in all $\mu$-XCT studies, is carefully controlling and improving the quality not only of the objects' scanning and slice images reconstructing processes, but also of the image processing steps following them (reduction of noise, ring artifacts, and beam hardening). The next essential steps are the 2D and/or 3D images enhancing preparing the segmentation processes, especially when there is no clear contrast between the different geomaterial phases. Consequently, at least visual expertvalid enhancing-segmentation procedures have to be selected for each case study by ranking the selected enhancing-segmentations, among a multiplicity of methods which are available today. In this case study, first, a visual, sufficiently robust, easy-to-implement, unambiguous, signal-processing-based, enhancing procedure will be selected, resulting from visually correlating and ranking the two algorithmic procedure results. One to segment the "TV" model and the other to enhance the "SR" model to prepare its segmentation step. With the use of this ranking it is possible, for the first time, not only to enhance the quality of the reconstructed images with a wide range of available procedures and decide almost automatically to select the enhancing method that best enables the segmentation step to be started, in order to better separate the existing significant parts of the signal (solid matrix components and/or voids) from the noise and other artefacts always existing in/on scanned and reconstructed images. The proposed solution was extensively tested on $\mu$-XCT scanned images of one sample of each sound and weathered sedimentary stone types (SR; MC; TV) [1], [3]. Those stones are also being investigated by the combined application of classic methods: Optical Microscopy, Scanning Electron Microscopy and Mercury Injection Porosimetry (MIP). In order to 
achieve future better qualitative and quantitative integrated models, it will be important to combine its nondestructive and 3D characteristics results with those of other 2D and 3D MIP models of those complex materials. Considering this approach and the results obtained may have profound implications and developments for more accurate image-based characterization of sedimentary geomaterials used in heritage objects, further qualitative/quantitative studies will follow. In particular the details of the enhancing and segmentation steps and the critical examination of the results are postponed to a following more extended article.

The authors acknowledge FCT projects: PEst-OE/CTE/UI0098/2011 and PEst- OE/CTE/UI0697/2011.

[1] Alves C, et al., Environmental Earth Science, 63, 1511-1527, 2010.

[2] A. Maurício, et al., Materials Science Forum, 636-637, 1306-1312, 2010.

[3] A. Maurício, et al. Environ Earth Sci, DOI 10.1007/s12665-013-2311-5, 2013.

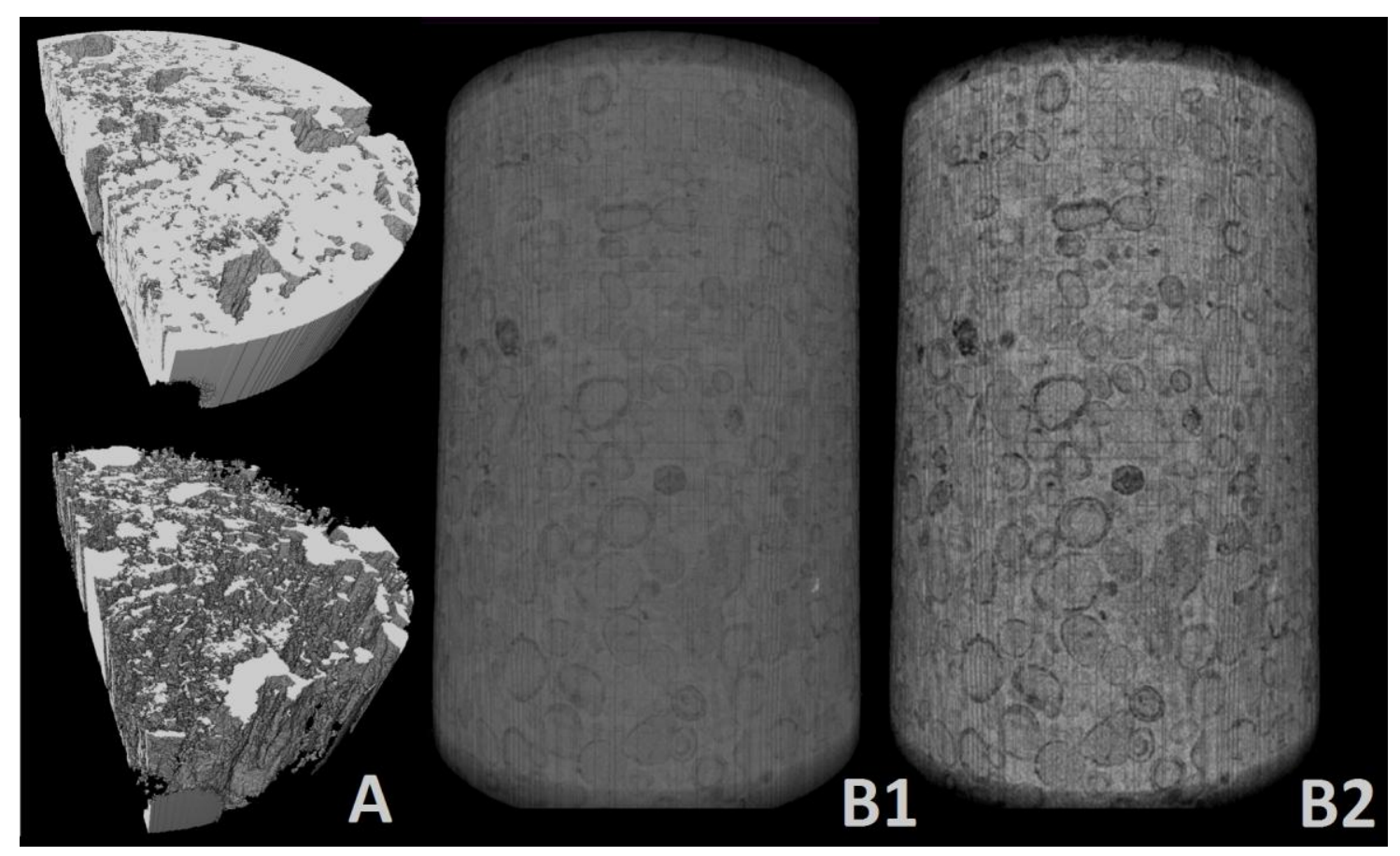

Figure 1 - Core samples diameter scales 5mm”. Pixel size resolution $4 \mu \mathrm{m}$. (A) - Two perspectives of two 3D virtual models resulting from part of the "Travertino" cylindrical core sample: reconstructed slice images; valid segmentation; rendering processes (Lower - Pores, Upper - Solid Matrix). (B) - One perspective of the studied "Semi-rijo" core sample. (B1) - 3D virtual model resulting from reconstructing and rendering. (B2) - The same model after a valid enhancement process preparing a valid segmentation step. Here the fossiliferous pelmicrosparite/grainstone nature of "Semi-rijo" is clearly shown in B. 